\title{
28 Research Suare \\ Malaria Data Analysis Using Routine Surveillance Data, Sunyani Municipality, Ghana, 2020
}

\author{
Abdul Gafaru Mohammed \\ University of Ghana School of Public Health \\ Christopher Sunkwa Tamal \\ Ghana Health Service \\ Magdalene Akos Odikro \\ University of Ghana School of Public Health \\ Delia Akosua Bandoh ( $\nabla$ deliabandoh@st.ug.edu.gh ) \\ University of Ghana School of Public Health https://orcid.org/0000-0002-0281-5321 \\ Charles Lwanga Noora \\ University of Ghana School of Public Health \\ Prince Romeo Quarshie \\ Ghana Health Service \\ Edwin Afari \\ University of Ghana School of Public Health \\ Ernest Kenu \\ University of Ghana School of Public Health
}

\section{Research}

Keywords: Malaria, Sunyani, Trend, Surveillance, Municipality, OPD, SMC, threshold

Posted Date: March 9th, 2021

DOI: https://doi.org/10.21203/rs.3.rs-168549/v2

License: (c) (i) This work is licensed under a Creative Commons Attribution 4.0 International License.

Read Full License 


\section{Abstract}

Introduction

Malaria is endemic in Ghana, accounting for about $40 \%$ of all OPD diagnosis. Data on malaria is routinely collected as part of the IDSR monthly reporting forms. Sunyani municipality recorded 56,540 malaria cases in 2016 with about $35 \%$ of the cases occurring in children under five years of age. We analyzed malaria surveillance data to identify the distribution of malaria cases by person, place and time and determine the timeliness and completeness of malaria report submission in the municipality.

Methods

We analyzed malaria surveillance data in Sunyani municipality reported between 2015 and 2019 from the District Health Management Information System II. We calculated morbidity and mortality rates of cases by person and estimated proportion of cases by sub-districts. We performed trend analysis and calculated disease threshold levels. We presented the findings using tables and figures.

Results

Of 639,361 malaria cases suspected, $93.5 \%(597,512 / 639,361)$ were tested, of which $49.4 \%$ $(295,458 / 597,512)$ was positive. Females accounted for $55.7 \%(164,436 / 295,458)$ of the morbidity. Children $<5$ years recorded the highest proportion, $29.9 \%(88,135 / 295,458)$ of cases in the municipality. The case fatality rate was $3.7 \%(18 / 484)$ and $1.4 \%$ (7/484) for persons under age five and over age five, respectively. Abesim $(6,276.03$ per 10,000) recorded the highest number of cases, and the least cases were in New Dormaa (1,890.97 per 10,000). The majority of malaria cases were in May and October of each year. Antwi-krom recorded the highest rates of completeness and timeliness of $41.9 \%$ and $40.0 \%$ respectively.

Conclusion

More females were diagnosed with malaria and children $<5$ years recorded the highest proportion of cases in the municipality. Abesim recorded the highest proportion of malaria cases during the period. High patterns of malaria transmissions occurred during months of high rainfalls. None of the subdistricts met the WHO target for timeliness and completeness of report submission. The NMCP should consider interventions such as SMC among children under five, in the Sunyani municipality.

\section{Background}

In 2018, 228 million cases of malaria were reported globally, with an estimated 405,000 malaria-related deaths. The WHO African Region accounted for $94 \%$ of all this death [1]. Particularly vulnerable to malaria are pregnant women and children under five years [2]. The illness claims a child's life every 2 minutes, making it the second-largest cause of death from infectious diseases in Africa [3]. About $67 \%$ $(272,000)$ of all malaria-related deaths in 2018 occurred in children under five years of age [1]. 
Malaria remains a hyperendemic disease posing a significant public health challenge in Ghana [4], [5]. It is the leading cause of morbidity and mortality despite the scale-up of new malaria preventive and control interventions [6], [7]. Furthermore, malaria ranks first among outpatient diagnoses, accounting for approximately $40 \%$ of all Out-Patient Department diagnoses [8]. According to the 2017 annual malaria report, the total number of malaria-related deaths in 2017 was 599, representing a decrease of about $54.6 \%$ over 1,264 malaria-related deaths in 2016 . Out of these malaria deaths, $54.6 \%$ (327/599) occurred among children under-5-years in 2017 compared to 46.7\% (590/1264) in 2016 [9].

Sunyani municipality recorded an upward trend of malaria cases from 43,467 in 2013 to 56,540 cases in 2016 , with about $35 \%$ of the cases occurring in children under five [11]. Malaria morbidity and mortality data in the municipality is collected through the passive surveillance approach. Even with the malaria surveillance system in the municipality, there has been limited epidemiological analysis of the data gathered to inform decision making on malaria preventive and control measures. We thus analyzed the five years data to determine the distribution of malaria cases by person, place and time and to determine the timeliness and completeness of malaria report submission in the municipality.

\section{Methods}

\section{Study design}

We analyzed malaria surveillance data in Sunyani municipality reported between 2015 and 2019 from the District Health Management Information System II. Malaria dataset from the system was reviewed and extracted for the analysis. We conducted the study from July 27 - September 05, 2020.

\section{Study Area.}

We conducted the study in the Sunyani Municipality. Sunyani municipality is one of the 260 Metropolitan, Municipal and District Assemblies (MMDAs) in Ghana. The projected 2019 population of the municipality was 151,378 [12]. About $18.5 \%$ of the total population are children $<5$ years old. The Sunyani municipality is in the semi equatorial climate zone. The median monthly temperatures range from $23^{\circ} \mathrm{C}$ $33^{\circ} \mathrm{C}$ with the lowest in August, and the highest observed in March and April. During the rainy seasons, relative humidity is high on average between $75 \%-80 \%$ and $70 \%-80 \%$ during the dry season. The municipality experiences double cycled rainfall, the primary rainy season runs from March to September with the minor being between October and December. During the rainy seasons, an increase in temperature, rainfall, and humidity may cause a proliferation of malaria-carrying mosquitoes, increasing malaria transmission. There are six sub-districts in the municipality: Sunyani Central, Abesim, New Dormaa, Ankwi-Krom, Penkwase and New Town Baakoniaba with 37 health facilities all reporting on malaria morbidity and mortality.

\section{Case definition of malaria}


Suspected Uncomplicated malaria: Any person with fever or history of fever within 24 hours; without signs of severe disease (vital organ dysfunction) is diagnosed clinically as uncomplicated malaria.

Suspected severe malaria: Any patient hospitalized with severe febrile disease with accompanying vital organ dysfunction diagnosed clinically [13].

Confirmed case of malaria: Occurrence of malaria illness/disease in a person in whom the presence of malaria parasites in the blood has been confirmed by parasitological testing [13].

\section{Data collection}

A data extraction tool was designed and employed for the extraction of malaria morbidity and mortality data from the DHIMS 2 platform. The variables extracted included; case status, age, sex, time of illness, place of residence, time of report submission and reports submitted.

\section{Data Analysis}

We used Microsoft Office Excel 2019 and Stata version 15.1 in analyzing quantitative data. Summary descriptive analysis of the data was done in the form of frequencies, averages, rates, thresholds, proportions and percentages. We used tables, charts and graphs to present the data.

In determining the proportion of confirmed malaria cases for all ages from 2015-2019: The malaria morbidity data was grouped by predetermined age groups and sex in the DHIMS 2 platform, that is $<28$ days, $1-11$ months, $1-4$ years, $5-9$ years, $10-14$ years, $15-17$ years, $18-19$ years, $20-34$ years, 35-49 years, 50-59 years, 60-69 years, and 70+. With the frequencies given for each age category in DHIMS 2, we estimated the relative frequencies or proportions for each age group. We performed the estimation by expressing the number of malaria cases (age-group) divided by total confirmed cases by $100 \%$.

In describing the distribution of malaria morbidity and mortality by sub-districts in the municipality, cases of malaria on DHIMS 2 were categorized on a sub-district basis. To determine the proportions of malaria cases in the various sub-districts in 2019 , we used the 2019 population distribution for the various subdistrict as the denominator and the 2019 case count from the sub-district as the numerator, per 10,000 population.

In assessing the trend of malaria morbidity and mortality in the municipality, we categorized the extracted malaria morbidity data based on time of occurrence (month and year) on Microsoft Office Excel. We drew a line graph using the absolute case counts for each of the months from 2015-2019. In determining the threshold levels for malaria in the municipality using the CUSUM-2 approach, we generated the mean number of cases for a preceding 7-month period, leaving a 2-month grace period, for each month. The standard deviations for the same period were derived and multiplied by 2 . The sum of the mean and two times the standard deviation gave the threshold level for each month. We calculated the threshold levels from September 2015 to December 2019. 
In assessing the timeliness and completeness of malaria data reporting, we extracted data on the number of reports submitted by a sub-district on time and the number of reports submitted in total by the same sub-district from the DHIMS 2 platform for timeliness and extracted data on the total number of reports submitted by a sub-district and the number of reports expected from the sub-district for completeness. The proportions resulting from this estimation were compared to the WHO target of $80 \%$ for timeliness and completeness of report submission [14].

\section{Ethical considerations}

Ghana Field Epidemiology and Laboratory Training Program (GFELTP) of the University of Ghana obtained permission from the Ghana Health Service to conduct malaria surveillance data analysis. The permission was through the National Malaria Control Programme (NMCP) as well as the Bono Regional Director of Health Services and the Sunyani municipal health directorate.

\section{Results}

\section{Distribution of malaria morbidity and mortality by person}

A total of 639,361 cases of malaria were suspected from the 2015-2019 period. Of the total cases suspected, $93.5 \%(597,512 / 639,361)$ were tested using microscopy and RDT methods. Over forty-nine per cent $(295,458 / 597,512)$ of the people tested were positive. About $5.4 \%(34,737 / 639,361)$ of the total suspected cases were treated without testing in the municipality.

Females accounted for approximately $55.7 \%(164,436 / 295,458)$ of the total confirmed cases. Malaria in children under age 5 accounted for $29.9 \%(88,135 / 295,458)$ of malaria burden. Uncomplicated malaria cases constituted $99.8 \%(294,974 / 295,458)$ of the confirmed cases with the remaining being severe malaria cases. Pregnant women consisted of $1.5 \%(2,387 / 164,436)$ of the total confirmed malaria cases among females. The case fatality rate among persons diagnosed with severe malaria was $3.7 \%(18 / 484)$ and $1.4 \%(7 / 484)$ for children under age five and over age five, respectively (Table 1$)$. 
Table 1

Age and sex distribution of malaria cases in Sunyani municipality, 20152019

\begin{tabular}{|lllll|}
\hline Variable & Under 5 & \multicolumn{3}{l|}{ Over 5 } \\
\hline & Frequency & Percentage & Frequency & Percentage \\
Sex & & & & \\
Male & 45080 & 15.3 & 85942 & 29.1 \\
Female & 43055 & 14.6 & 121381 & 41.1 \\
Classifications & & & & \\
Uncomplicated & 88030 & 29.8 & 206944 & 70.0 \\
Severe Malaria & 105 & .03 & 379 & .13 \\
\hline Disease outcome & & & & \\
\hline Died & 18 & 3.7 & 7 & 1.4 \\
\hline Recovered & 87 & 18.0 & 372 & 76.9 \\
\hline Pregnancy status & & & & \\
\hline Pregnant & & & 2387 & 1.5 \\
\hline Not pregnant & 43055 & 26.2 & 118994 & 72.4 \\
\hline
\end{tabular}

\section{Distribution of confirmed cases of malaria by sub-districts in the Sunyani municipality}

In 2019, the proportion of malaria was high (6,276.03 per 10,000) among residents of Abesim, and the least $(1,890.97$ per 10,000) was recorded among residents of New Dormaa sub-district (Fig. 2).

\section{Distribution of confirmed cases of malaria by the time of occurrence in the municipality}

Cases of malaria were recorded in all months between January 2015 and December 2019. The highest number (8,067 persons) of cases over the period were recorded in October 2018 and the lowest $(2,524$ persons) in February 2015 (Fig. 2). With the threshold determined, the highest threshold level was in May 2019 and the lowest level in June 2016. The months of May and October 2016 and October of 2018, saw the number of malaria cases exceed the threshold level. In May 2018, the number of cases was almost equal to the threshold level (Fig. 2). 


\section{Timeliness and completeness of malaria report submission in the Sunyani municipality}

For the five years under review, Antwi-krom recorded the highest completeness and timeliness of $41.9 \%$ and $40.0 \%$ respectively. Sunyani central recorded the least completeness and timeliness of $22.1 \%$ and $20.1 \%$, respectively (Fig. 3).

\section{Discussion}

We set out to analyze the five-year data of the Sunyani municipality from 2015 to 2019 to identify the distribution of malaria cases by person, place and time and to determine the timeliness and completeness of malaria report submission in the municipality.

In analyzing the distribution of malaria cases in the municipality by person, approximately $55.7 \%$ of the persons diagnosed with malaria were females. This could be explained by the higher proportion of females than males in the municipality's general population [15]. This is consistent with the findings of a study conducted in Manicaland province Zimbabwe, where 52.5\% of cases confirmed were females [16]. The similarity in result could likely be explained by the Manicaland province and Sunyani municipality's similar population structure. Each of them has over $52 \%$ of its population being females [15], [17]. Despite the higher malaria cases among females in the general population, we found that the prevalence of malaria was higher among male children than female children under five years. This finding was consistent with similar studies in Ghana and Kenyan [18], [19]. The authors attributed the disparity to female children being less biologically vulnerable to infectious diseases than their male counterparts.

About one-third of confirmed cases of malaria were in children under five years. This is similar to what Bajoga et al., (2019) reported in Kaduna, Nigeria. Malaria cases were fewer among children less than one year. This may be partly explained by the increased push to combat malaria through a combination of interventions such as enhanced ITN coverage, improved antenatal care and IPTP-SP uptake [21]. Among the children less than a year old, neonates had the lowest proportion of malaria infections. This may be because, during pregnancy, neonates obtained antibodies from their mothers [18]. Furthermore, a study conducted by Stephens et al., (2017) found that pregnant women exposed to complete IPTp-SP had better protection with reduced placental parasitemia while serving as a buffer for their neonates.

The Sunyani municipality recorded a high malaria case fatality rate of $5.1 \%$ with about $3.4 \%$ fatality rate among children under five years of age. The high fatality rate among the under five years could be attributed to the delay in getting these children to a health facility for medical care before the onset of complications. However, this finding is inconsistent with the malaria case fatality rate reported by the National Malaria Control Program for the year 2017. The difference in the fatality rate could be attributed to the difference in the population size under surveillance. 
The Abesim sub-district registered the highest malaria burden in the municipality from 2015-2019. This could be explained by the Ghana Water Company water treatment plant's presence in the area that could serve as a breeding ground for mosquitoes and hence the high transmission rate. This finding is similar to a work conducted in Central Ethiopia, where villages with irrigation dams were found to have 3.6 times increased odds of malaria transmission compared to villages without irrigation plants [23]. We ruled out other factors such as high reporting rate by facilities in the area or population proportion compared to the other sub-districts as possible factors accounting for the high transmission in the area.

In the municipality, there was a seasonal variation to the transmissions of malaria over the five years. Cases of malaria were more pronounced in May, June and October of every year for the period. This could be explained by the municipality's rainfall pattern, where the major rainfalls occur from March to September and the minor one between October and December each year. This result is consistent with the findings of a study conducted in Limpopo to assess the climatic variables and malaria transmission; they reported that monthly rainfall was the main predictor of malaria transmission [24]. However, this is inconsistent with the findings of a similar study conducted in northeastern rural Benin where the authors' associated high incidence of malaria with dry or drought seasons of the year [25]. The disparity in this finding could be explained by the difference in the climate of the areas [12], [25]. The municipality experienced an excess case count above the threshold levels in May and October 2016 and October of 2018. This could be suggestive of an outbreak of malaria. This is consistent with the findings of Lechthaler et al., (2019) \& Ogwang et al., (2018) where they detected malaria epidemics from trend analysis of malaria data in Kitgum district Uganda and DR Congo. However, in the case of Sunyani municipality, certain factors needed to be ruled out to confirm the status of the increase, whether it was a true or false increase. These factors include; batch reporting of cases by facilities, changes in reporting practices of surveillance officers, errors in the reporting of cases and improvements in diagnostic methods for malaria. Also, malaria being an episodic disease could have resulted in over-reporting, which was suggestive of the outbreak.

For the five years under review, the timeliness and completeness of malaria report submission by the subdistricts in the municipality was low with none of the sub-districts meeting the WHO target of $80 \%$ for completeness and timeliness of IDSR monthly reports submission. The poor timeliness of report submission is consistent with the findings of a study conducted in Malawi [14]. In contrast to this finding, studies conducted in northern Ghana and Uganda reported reasonable rates on timeliness and completeness of IDSR monthly report submission [28], [29].

There were a few limitations to this study. Firstly, malaria is an episodic disease, and the number of cases reported could have been influenced by over-reporting in the municipality. Secondly, some private health facilities in the municipality were not reporting malaria morbidity and mortality data; this could have affected the municipality's actual burden. However, we kept in many efforts to retrieve data from private health facilities and compare hardcopies IDSR forms and hospital registers to the records in the DHIMS 2 to make the findings of this analysis significant and informing. 


\section{Conclusion}

The occurrence of malaria in the municipality varied according to age and gender with children $<5$ and females recording a higher proportion. Abesim sub-district recorded a high proportion of malaria cases in 2019. There was seasonal variation in the transmission of malaria in the municipality during the period under review. None of the sub-districts met the WHO target for timeliness and completeness of report submission. The NMCP should consider interventions such as SMC among children $<5$ years and indoor residual spraying (IRS) in the Sunyani municipality. The municipal health directorate should strengthen education in the municipality on malaria control and treatment adherence.

\section{List Of Abbreviations}

CDC Center for Disease Control \& Prevention

DHIMS District Health Information Management Systems

DHMT District Health Management Team

GFELTP Ghana Field Epidemiology and Laboratory Training Programme

GHS Ghana Health Service

HIO Health Information Officer

IDSR Integrated Disease Surveillance and Response

IPTp Intermittent Preventive Treatment of Pregnant women

IRS Indoor Residual Spraying

ITNs Insecticide Treated Nets

$\mathrm{MOH}$ Ministry of Health

NMCP National Malaria Control Programme

PCR Polymerase Chain Reaction

RDT Rapid Diagnostic Test

SMC Seasonal Malaria Chemoprevention

WHO World Health Organization

\section{Declarations}




\section{Ethical declarations}

Ghana Field Epidemiology and Laboratory Training Program (GFELTP) of the University of Ghana obtained permission from the Ghana Health Service to conduct malaria surveillance data analysis. The permission was through the National Malaria Control Programme (NMCP) and the Bono Regional Director of Health Services and the Sunyani municipal health directorate.

\section{Consent for publication}

Not applicable

\section{Availability of dataset or materials}

The dataset supporting the conclusions of this article is included within the article (and its additional file(s))

\section{Competing interest}

The authors declared that they had no competing interest

\section{Funding}

Not applicable

\section{Contributions}

AGM, CT conceptualized the study, implemented data acquisition, performed the analysis and drafted the initial manuscript. DB, MAO, CLN, PRQ, EA, EK edited and reviewed the manuscript. All authors read and approved the final manuscript

\section{Acknowledgement}

We are grateful to the NMCP for their support and the Sunyani Municipal health directorate for providing us with the dataset

\section{References}

1. Global Malaria Programme: WHO Global. "World malaria report 2019," WHO Regional Office for Africa, 2019. [Online]. Available: https://www.who.int/news-room/fact-sheets/detail/malaria.

2. Afoakwah C, Deng X, Onur I, "Malaria infection among children under-five: The use of large-scale interventions in Ghana," BMC Public Health, vol. 18, no. 1, Apr. 2018.

3. Duguma FK, "Assessment of Knowledge, Attitude and Practice on Insecticide Treated Net Utilization towards Malaria Prevention among Ethiopian Army members of 24th Division, 2016," Int. J. Environ. Sci. Nat. Resour., vol. 21, no. 3, Sep. 2019. 
4. Asare EO, Amekudzi LK, "Assessing climate driven malaria variability in Ghana using a regional scale dynamical model," climate, vol. 5, no. 1, 2017.

5. NMCP. “National Malaria Control Programme Ghana Malaria Programme Review," no. June. p. 24, 2013.

6. Ameme DK, Afari EA, Nyarko KM, Malm KL, Sackey S, Wurapa F, “Direct observation of outpatient management of malaria in a rural ghanaian district," Pan Afr. Med. J., vol. 19, Dec. 2014.

7. Nkegbe PK, Kuunibe N, Sekyi S, Aye G. Poverty and malaria morbidity in the Jirapa District of Ghana: A count regression approach. Cogent Econ Financ. Jan. 2017;5(1):1293472.

8. 2015 A report NMCP. "2015 ANNUAL REPORT NATIONAL MALARIA CONTROL Centre for Health Information Management," pp. 1-70, 2016.

9. GHS. "2017 Annual Report National Malaria Control Programme," Adv. Cancer Res., 2018.

10. PMI “US.. PRESIDENT’ S MALARIA INITIATIVE GHANA Malaria Operational Plan FY 2020,” 2020.

11. A. C. P. and Effah-Acheampong A-WK, Edwin J, Afari A. S.O. Sackey Ameme Donne, Kenu Ernest, Kumalor George, "Malaria surveillance system evaluation, sunyani municipality, Ghana-2017 EffahAcheampong. J Infect Dis Ther. 2018;06:2332-0877.

12. GSS. "Sunyani Municipal (Municipal District, Ghana) - Population Statistics, Charts, Map and Location," 2020. [Online]. Available: https://www.citypopulation.de/en/ghana/admin/bono/0703_sunyani_municipal/. [Accessed: 18Sep-2020].

13. Ghana IDSR. "Technical Guidelines Integrated Disease Surveillance \& Response Ghana, 2," 2017.

14. Joseph T-S, Id W, Kagoli M, Kaasbøll JJ, Bjune GA. "Integrated Disease Surveillance and Response (IDSR) in Malawi: Implementation gaps and challenges for timely alert," 2018.

15. SMA “ASSEMBLYFOR. 2019 PROGRAMME BASED BUDGET ESTIMATES FOR 2019-2022 COMPOSITE BUDGET REPUBLIC," 2017.

16. Mutsigiri F, et al., "Malaria morbidity and mortality trends in Manicaland province, Zimbabwe, 20052014," Pan Afr. Med. J., vol. 27, 2017.

17. ZIMSTAT. "MANICALAND PROVINCE DISTRICT POPULATION PROJECTIONS REPORT ii,” 2020.

18. Nyarko SH, Cobblah A, "Sociodemographic Determinants of Malaria among Under-Five Children in Ghana," vol. 2014, 2014.

19. Sultana M, Sheikh N, Mahumud RA, Jahir T, Islam Z. "Prevalence and associated determinants of malaria parasites among Kenyan children," pp. 1-9, 2017.

20. Bajoga UA, et al. Trend of malaria cases in Kaduna State using routine surveillance data, 20112015. Pan Afr Med J. 2019;32:8.

21. Ibrahim H, Maya ET, Issah K, Apanga PA, Bachan EG, Noora CL, "Factors influencing uptake of intermittent preventive treatment of malaria in pregnancy using sulphadoxine pyrimethamine in sunyani municipality, Ghana," Pan Afr. Med. J., vol. 28, Oct. 2017. 
22. Stephens JK, et al. Effect of IPTp on Plasmodium falciparum antibody levels among pregnant women and their babies in a sub-urban coastal area in Ghana. Malar J. 2017;16(1):1-10.

23. Kibret S, Wilson GG, Tekie $H$, Petros $B$, "Increased malaria transmission around irrigation schemes in Ethiopia and the potential of canal water management for malaria vector control," Malar. J., vol. 13, no. 1, Sep. 2014.

24. Adeola AM, et al., "Climatic variables and malaria morbidity in mutale local municipality, South Africa: A 19-year data analysis," Int. J. Environ. Res. Public Health, vol. 14, no. 11, Nov. 2017.

25. Govoetchan R, et al., "Evidence for perennial malaria in rural and urban areas under the Sudanian climate of Kandi, Northeastern Benin," Parasites and Vectors, vol. 7, no. 1, Feb. 2014.

26. Ogwang R, Akena G, Yeka A, Osier F, Idro R. The 2015-2016 malaria epidemic in Northern Uganda; What are the implications for malaria control interventions? Acta Trop. Dec. 2018;188:27-33.

27. Lechthaler F, et al., "Trends in reported malaria cases and the effects of malaria control in the Democratic Republic of the Congo," PLoS One, vol. 14, no. 7, Jul. 2019.

28. Adokiya MN, Awoonor-Williams JK, Barau IY, Beiersmann C, Mueller O. "Evaluation of the integrated disease surveillance and response system for infectious diseases control in northern Ghana," BMC Public Health, vol. 15, no. 1, Feb. 2015.

29. Masiira B, et al., "Evaluation of integrated disease surveillance and response (IDSR) core and support functions after the revitalization of IDSR in Uganda from 2012 to 2016," BMC Public Health, vol. 19, no. 1, Jan. 2019.

\section{Figures}




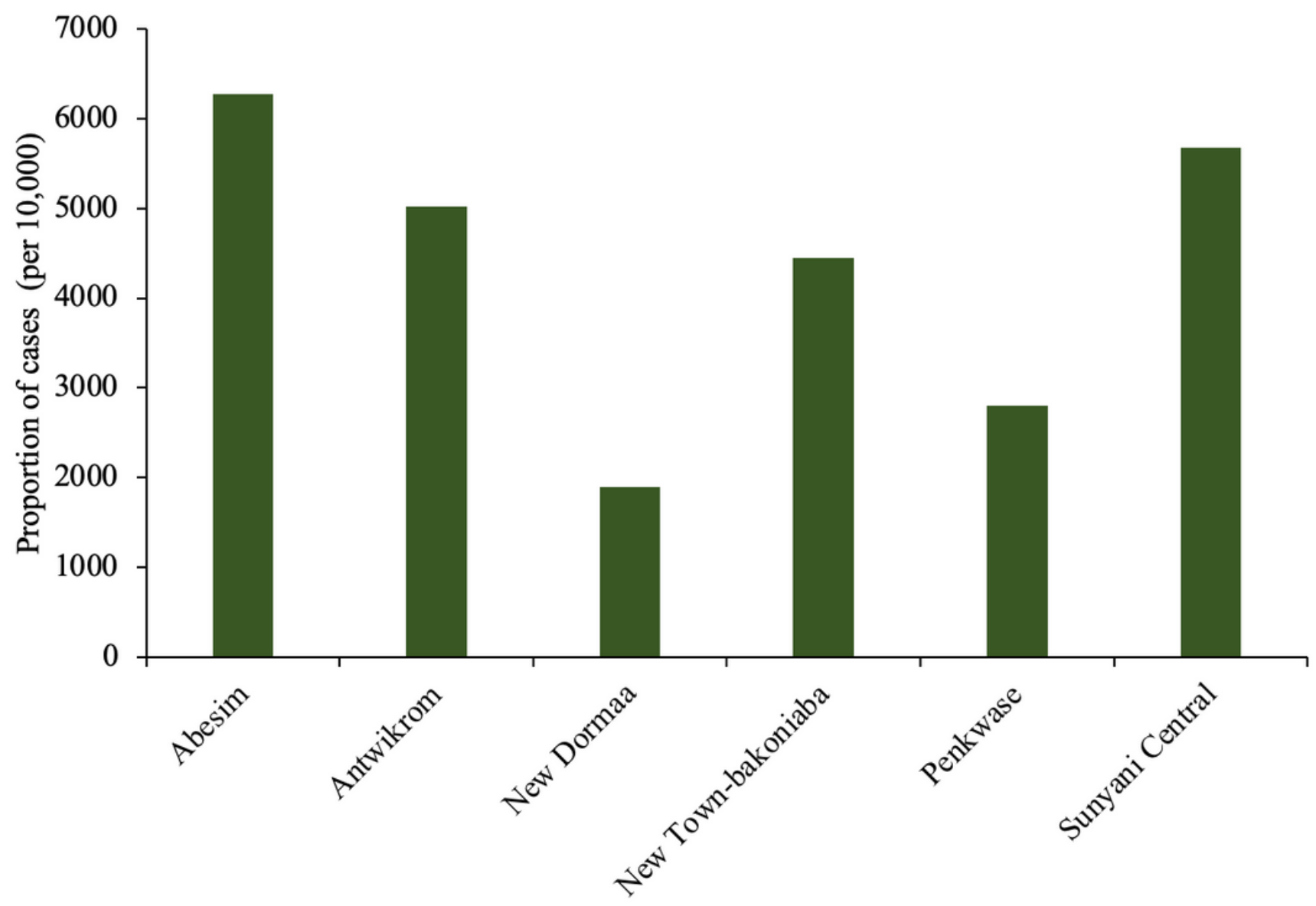

Sub-district

Figure 1

Sub-district distribution of confirmed malaria cases, 2019 


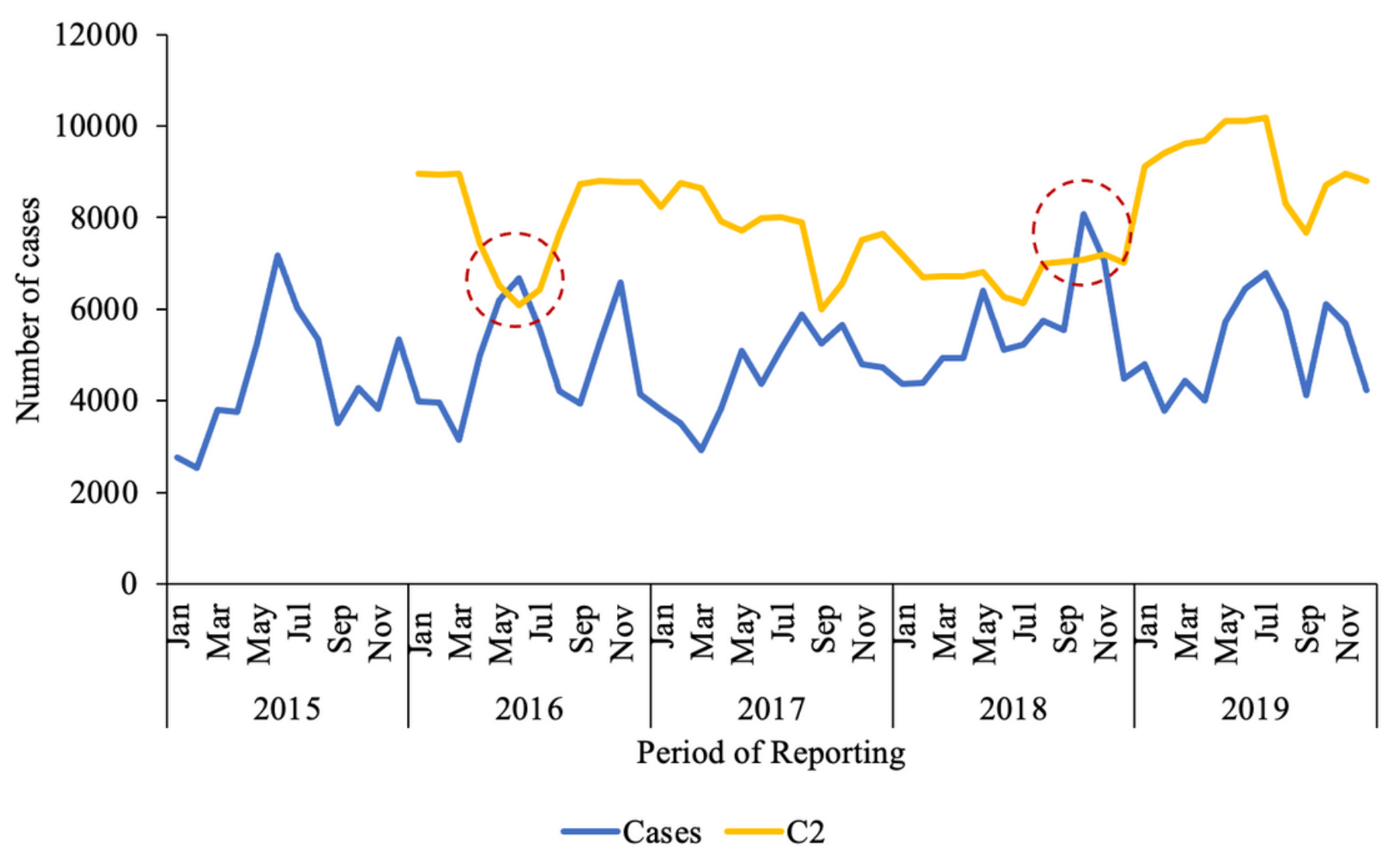

Figure 2

Confirmed malaria cases reported and malaria threshold levels (C2) for Sunyani municipality, January 2015 to December 2019 


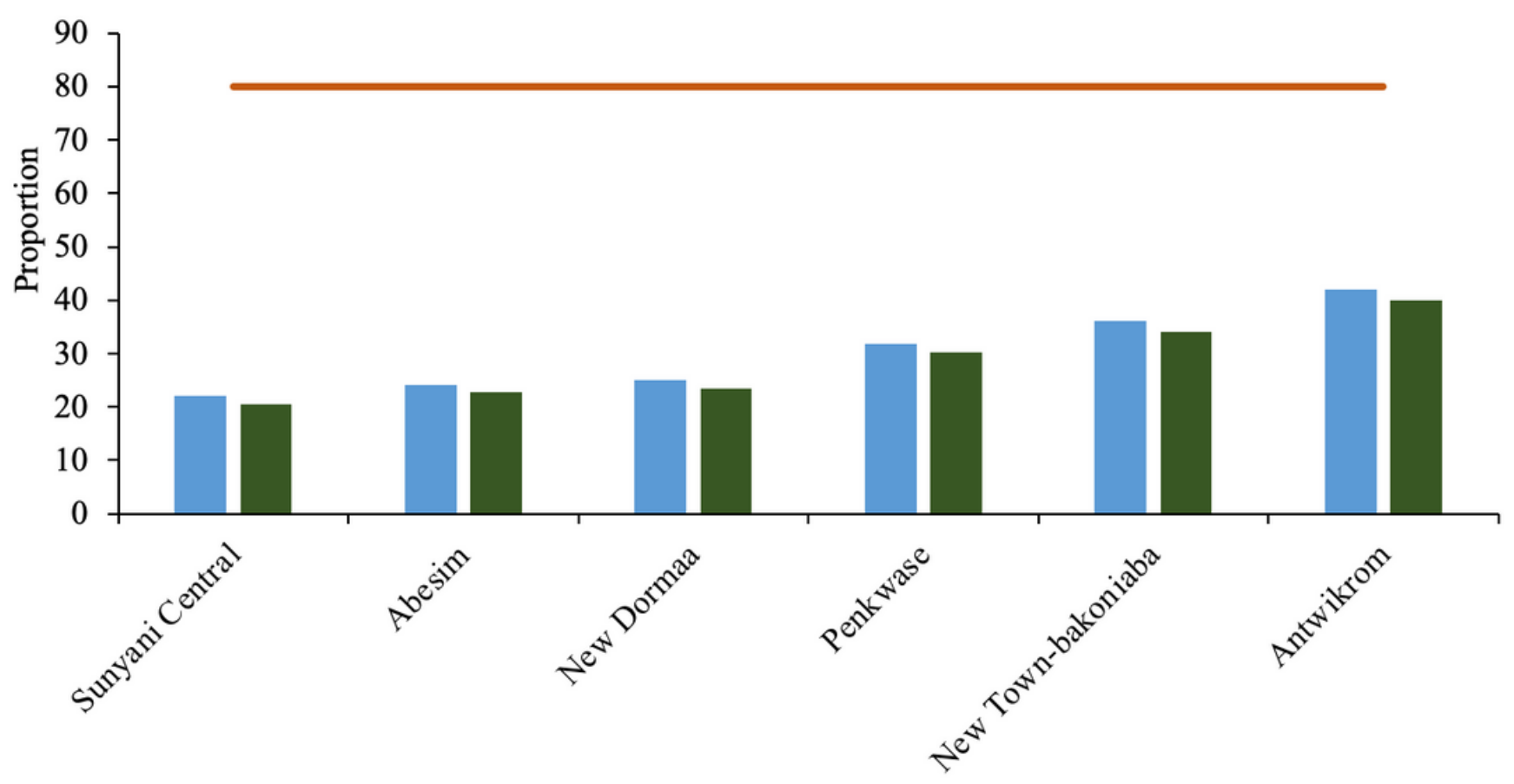

Sub-District

\section{Figure 3}

Timeliness and completeness of malaria data reporting by sub-districts, 2015-2019

\section{Supplementary Files}

This is a list of supplementary files associated with this preprint. Click to download.

- Datasetformanuscript.xlsx 